KEK-TH-349

KEK preprint 92-169

April, 1993

\title{
Chiral Perturbation to One Loop Including the $\rho$ Meson
}

\author{
MASAHARU TANABASHI* \\ National Laboratory for High Energy Physics (KEK) \\ Tsukuba, Ibaraki 305, Japan
}

\begin{abstract}
We formulate the chiral perturbation theory at the one loop level in the effective lagrangian including the $\rho$ meson as a dynamical gauge boson of a hidden local symmetry(HLS). The size of radiative correction to the phenomenological parameter $a$ of HLS is estimated to be about $10 \%$. The complete list of $\mathcal{O}\left(E^{4}\right)$ terms is given and the one loop counter terms are determined explicitly in the $N$ flavor model. We also obtain matching conditions to the conventional chiral perturbation of Gasser and Leutwyler in the chiral limit in a renormalization scale independent manner. We find that Gasser-Leutwyler's estimates for $L_{9,10}$ are saturated by $\rho$ and its one loop contributions without introducing non-minimal couplings of $\pi-\rho$ system, suggesting the absence of the tree level $a_{1}$ meson contributions.
\end{abstract}

Chiral perturbation theory (ChPT) [1, [ 2] is a framework to describe low energy nature of the spontaneous chiral symmetry breaking in QCD. It gives a systematic low energy expansion of QCD amplitudes in terms of the number of derivatives appearing in the effective lagrangian. Based on the well-known isomorphism between the electroweak symmetry breaking and the spontaneous chiral symmetry breaking in QCD, ChPT becomes very popular also in the physics of the Higgs sector. Actually, the method of chiral lagrangian offers a perspective view in the analysis of precision tests of the Higgs sector 3 .

The effective lagrangian (chiral lagrangian) written in terms of the Nambu-Goldstone $(\mathrm{NG})$ boson field with the lowest order of derivatives is $\mathcal{O}\left(E^{2}\right)\left(\equiv \mathcal{O}\left(\partial^{2}\right)\right)$, which reproduces results of the low energy theorems. Taking account of the $\mathcal{O}\left(E^{4}\right)$ terms and the

${ }^{*}$ E-mail address: tanabash@theory.kek.jp 
one loop corrections, we can extract further information from the chiral symmetry [1, 2] than those from the low energy theorems.

It is evident, however, that the ChPT cannot be applied at the scale of the mass of the $\rho$ meson (the lightest non-NG boson) in QCD. We need to introduce an explicit degree of freedom corresponding to the $\rho$ meson so as to make the effective lagrangian valid at the scale of its mass. Bando, Kugo, Uehara, Yamawaki and Yanagida (BKUYY) [4 constructed the most successful effective lagrangian of the $\pi$ - $\rho$ system based on the idea of hidden local symmetry. This lagrangian, being identical with Weinberg's model|5] in the unitary gauge, describes successfully some phenomenological properties in the $\pi$ - $\rho$ system such as the $\rho$ coupling universality[6] (and thus, the $\rho$ dominance 6]) and the KSRF relation [7] by choosing its phenomenological parameter $a=2$. The "vector limit" lagrangian [8] can be also considered as a special case of the model at $a=1$.

The BKUYY lagrangian has been applied to various processes at tree level. Analyses of kaon decays in the BKUYY lagrangian have been performed at tree level with a fixed (non-running) parameter $a[9]$. The effect of the $\rho$ meson on the Gasser-Leutwyler parameters $L_{i}$ [2] of the conventional ChPT is also investigated [10]. The lagrangian is known as the BESS model when it is applied to the Higgs sector [11]. Those tree level analyses, however, leave uncertainties from the radiative corrections. On the other hand, the analyses at the one loop level have been dealt with a few papers: Cvetič and Kögerler 12] calculated divergent coefficients in the self-interactions of the gauge fields in the BESS model. Harada and Yamawaki 13 showed that the low energy theorem 14] of the hidden local symmetry (HLS) is maintained at one loop in the Landau gauge.] However, those one loop level calculations are not sufficient to estimate the size of radiative corrections in the BKUYY model of $\pi$ - $\rho$ system.

In this paper, we address this problem by formulating the ChPT of the BKUYY effective lagrangian [4] in a systematic manner at one loop. The complete list of the $\mathcal{O}\left(E^{4}\right)$ terms not restricted to the self-interaction of gauge fields is obtained and the one loop counter terms are determined explicitly in the $S U(N)$ model. The size of the radiative correction to the phenomenological parameter $a$ is estimated to be about $10 \%$. We also obtain several matching conditions to the conventional ChPT without the $\rho$ meson in a renormalization scale independent manner. Remarkably enough, the one loop effect of the $\pi-\rho$ system is shown to imitate the tree level contribution 10 from the $a_{1}$ meson in the Gasser-Leutwyler parameter $L_{10}$. We find that the Gasser-Leutwyler estimates[2] for $L_{9,10}$ are saturated by $\rho$ and its one loop contributions without introducing non-minimal $\mathcal{O}\left(E^{4}\right)$ couplings in $\pi$ - $\rho$ system.

Let us start with a quick review of the BKUYY lagrangian 4 . BKUYY decomposed the pion field $U=\exp \left(2 i T^{a} \pi^{a}\right)$, transforming $U \rightarrow g_{L} U g_{R}^{\dagger}$ under $S U(N)_{L} \times S U(N)_{R}$ into two parts,

$$
U=\xi_{L}^{\dagger} \xi_{R}
$$

\footnotetext{
1 Although the low energy theorem of HLS holds only at the off-shell of the $\rho$ meson and is not directly related to the physical amplitudes, the phenomenological success indicates that the low energy theorem is smoothly extrapolated to the on-shell of the $\rho$ meson.
} 
Arbitrariness in this decomposition can be regarded as a local symmetry (hidden local symmetry, HLS):

$$
\xi_{L, R} \rightarrow h(x) \xi_{L, R}, \quad h(x) \in S U(N)_{\mathrm{HLS}} .
$$

This hidden local "symmetry" is redundant and nothing to do with the $\pi$ system at this level. By introducing a kinetic term for the gauge field associated with the HLS, however, it becomes a dynamical degree of freedom which may be identified as the $\rho$ meson.

This idea leads us to an effective lagrangian for the $\pi$ - $\rho$ system:

$$
\mathcal{L}=f^{2} \operatorname{tr}\left(\hat{\alpha}_{\mu \perp} \hat{\alpha}_{\perp}^{\mu}\right)+\frac{f^{2}}{4} \operatorname{tr}\left(\hat{\chi}+\hat{\chi}^{\dagger}\right)+a f^{2} \operatorname{tr}\left(\hat{\alpha}_{\mu \|} \hat{\alpha}_{\|}^{\mu}\right)-\frac{1}{2 g^{2}} \operatorname{tr}\left(V_{\mu \nu} V^{\mu \nu}\right)
$$

where $\hat{\alpha}_{\mu \perp}$ and $\hat{\alpha}_{\mu \|}$ are defined by

$$
\hat{\alpha}_{\perp}^{\mu} \equiv-\frac{i}{2}\left[\left(D^{\mu} \xi_{L}\right) \xi_{L}^{\dagger} \mp\left(D^{\mu} \xi_{R}\right) \xi_{R}^{\dagger}\right]
$$

The vector field $V_{\mu}$ is identified with the $\rho$ meson and we have introduced external fields $\mathcal{L}_{\mu}, \mathcal{R}_{\mu}, s$ and $p$ in an analogous manner to Gasser-Leutwyler [2]:

$$
\begin{aligned}
D_{\mu} \xi_{L} & \equiv \partial_{\mu} \xi_{L}-i V_{\mu} \xi_{L}+i \xi_{L} \mathcal{L}_{\mu} \\
D_{\mu} \xi_{R} & \equiv \partial_{\mu} \xi_{R}-i V_{\mu} \xi_{R}+i \xi_{R} \mathcal{R}_{\mu} \\
\hat{\chi} & \equiv 2 b \xi_{L}(s+i p) \xi_{R}^{\dagger}
\end{aligned}
$$

The constant $b$ measures the quark pair condensate in the chiral limit. The first two terms then correspond to the conventional chiral lagrangian at $\mathcal{O}\left(E^{2}\right)$, while the last two terms are the mass and the kinetic terms of the $\rho$ meson. It should be stressed here that the $\rho$ meson acquires its mass $\left(m_{\rho}^{2}=a g^{2} f^{2}\right)$ through the Higgs mechanism in the present model.

Now, we are ready to discuss a systematic expansion of the $\pi$ - $\rho$ amplitudes based on the lagrangian (3). We first study rules of counting the orders in the effective lagrangian. Since the external vector fields $\left(\mathcal{L}_{\mu}, \mathcal{R}_{\mu}\right)$ couple to $\xi$ as gauge connections, they should be balanced with a derivative in the order counting. The $\rho$ meson field $V_{\mu}$ is assigned $\mathcal{O}(E)$ for the same reason. The order of $\hat{\chi}$ is determined as $\mathcal{O}\left(E^{2}\right)$, since it produces the pion mass which should be balanced with $p^{2}$ in the propagator. It should be stressed that we need to assign $\mathcal{O}(E)$ to the coupling $g$ in order for the kinetic term of $\rho$ to be $\mathcal{O}\left(E^{2}\right)$. This assignment implies that the effective lagrangian is expanded in terms of the mass of the vector meson.

We next construct the most general effective lagrangian with $\mathcal{O}\left(E^{4}\right)$. It is convenient to make a list of operators having homogeneous transformation in the HLS. It is easy to show that

$$
\hat{\alpha}_{\mu \perp}, \quad \hat{\alpha}_{\mu \|}
$$


are the only operators in this class at the lowest order $(\mathcal{O}(E))$. In addition to the covariant derivatives of Eq.(6), there exist operators in higher orders, e.g.,

$$
V_{\mu \nu}, \quad \hat{\mathcal{V}}_{\mu \nu}, \quad \hat{\mathcal{A}}_{\mu \nu}, \quad \hat{\chi}
$$

where $V_{\mu \nu}$ is the field strength of the $\rho$ field and $\hat{\mathcal{V}}_{\mu \nu}$ and $\hat{\mathcal{A}}_{\mu \nu}$ are defined as

$$
\begin{aligned}
\hat{\mathcal{V}}_{\mu \nu} & \equiv \frac{1}{2}\left[\xi_{R} \mathcal{R}_{\mu \nu} \xi_{R}^{\dagger}+\xi_{L} \mathcal{L}_{\mu \nu} \xi_{L}^{\dagger}\right] \\
\hat{\mathcal{A}}_{\mu \nu} & \equiv \frac{1}{2}\left[\xi_{R} \mathcal{R}_{\mu \nu} \xi_{R}^{\dagger}-\xi_{L} \mathcal{L}_{\mu \nu} \xi_{L}^{\dagger}\right]
\end{aligned}
$$

with $\mathcal{R}_{\mu \nu}$ and $\mathcal{L}_{\mu \nu}$ being the field strength of $\mathcal{R}_{\mu}$ and $\mathcal{L}_{\mu}$, respectively.

The most general lagrangian at $\mathcal{O}\left(E^{4}\right)$ can now be constructed by taking a trace of products of those operators and the covariant derivatives of $\hat{\alpha}_{\mu \perp, \|}$. It should be, however, noticed that the anti-symmetric combination of the covariant derivatives of $\hat{\alpha}_{\mu \perp, \|}$ can be expressed in terms of other operators:

$$
\begin{aligned}
D_{\mu} \hat{\alpha}_{\nu \perp}-D_{\nu} \hat{\alpha}_{\mu \perp} & =i\left[\hat{\alpha}_{\mu \|}, \hat{\alpha}_{\nu \perp}\right]+i\left[\hat{\alpha}_{\mu \perp}, \hat{\alpha}_{\nu \|}\right]-\hat{\mathcal{A}}_{\mu \nu} \\
D_{\mu} \hat{\alpha}_{\nu \|}-D_{\nu} \hat{\alpha}_{\mu \|} & =i\left[\hat{\alpha}_{\mu \|}, \hat{\alpha}_{\nu \|}\right]+i\left[\hat{\alpha}_{\mu \perp}, \hat{\alpha}_{\nu \perp}\right]+\hat{\mathcal{V}}_{\mu \nu}-V_{\mu \nu}
\end{aligned}
$$

Thus, it is sufficient to consider the symmetric combinations:

$$
D_{\mu} \hat{\alpha}_{\nu \perp, \|}+D_{\nu} \hat{\alpha}_{\mu \perp, \|}-\frac{1}{2} g_{\mu \nu} D_{\rho} \hat{\alpha}_{\perp, \|}^{\rho}, \quad D_{\mu} \hat{\alpha}_{\perp, \|}^{\mu}
$$

The independent terms in $\mathcal{O}\left(E^{4}\right)$ lagrangian can be further reduced by using the equations of motion:

$$
\begin{aligned}
D_{\mu} \hat{\alpha}_{\perp}^{\mu} & =i(1-a)\left[\hat{\alpha}_{\mu \|}, \hat{\alpha}_{\perp}^{\mu}\right]+i\left(\hat{\chi}-\hat{\chi}^{\dagger}\right)+\mathcal{O}\left(E^{4}\right), \\
D_{\mu} \hat{\alpha}_{\|}^{\mu} & =\mathcal{O}\left(E^{4}\right), \\
D_{\mu} V^{\mu \nu} & =g^{2} a f^{2} \hat{\alpha}_{\|}^{\nu}+\mathcal{O}\left(E^{4}\right) .
\end{aligned}
$$

After a short algebra, we find the general form of $\mathcal{O}\left(E^{4}\right)$ lagrangian with the even intrinsic parity:

$$
\mathcal{L}_{4}=\mathcal{L}_{4}^{w}+\mathcal{L}_{4}^{x}+\mathcal{L}_{4}^{y}+\mathcal{L}_{4}^{z}
$$

where

$$
\begin{aligned}
\mathcal{L}_{4}^{w}= & w_{1} \operatorname{tr}\left(\hat{\alpha}_{\perp}^{\mu} \hat{\alpha}_{\mu \perp}\left(\hat{\chi}+\hat{\chi}^{\dagger}\right)\right)+w_{2} \operatorname{tr}\left(\hat{\alpha}_{\perp}^{\mu} \hat{\alpha}_{\mu \perp}\right) \operatorname{tr}\left(\hat{\chi}+\hat{\chi}^{\dagger}\right) \\
& +w_{3} \operatorname{tr}\left(\hat{\alpha}_{\|}^{\mu} \hat{\alpha}_{\mu \|}\left(\hat{\chi}+\hat{\chi}^{\dagger}\right)\right)+w_{4} \operatorname{tr}\left(\hat{\alpha}_{\|}^{\mu} \hat{\alpha}_{\mu \|}\right) \operatorname{tr}\left(\hat{\chi}+\hat{\chi}^{\dagger}\right)+w_{5} \operatorname{tr}\left(\left[\hat{\alpha}_{\perp}^{\mu}, \hat{\alpha}_{\mu \|}\right]\left(\hat{\chi}-\hat{\chi}^{\dagger}\right)\right) \\
& +w_{6} \operatorname{tr}\left(\left(\hat{\chi}+\hat{\chi}^{\dagger}\right)^{2}\right)+w_{7}\left(\operatorname{tr}\left(\hat{\chi}+\hat{\chi}^{\dagger}\right)\right)^{2} \\
& +w_{8} \operatorname{tr}\left(\left(\hat{\chi}-\hat{\chi}^{\dagger}\right)^{2}\right)+w_{9}\left(\operatorname{tr}\left(\hat{\chi}-\hat{\chi}^{\dagger}\right)\right)^{2} \\
\mathcal{L}_{4}^{x}= & x_{1} m_{\rho}^{2} \operatorname{tr}\left(\hat{\alpha}_{\mu \perp} \hat{\alpha}_{\perp}^{\mu}\right)+x_{2} m_{\rho}^{2} \operatorname{tr}\left(\hat{\alpha}_{\mu \|} \hat{\alpha}_{\|}^{\mu}\right)+x_{3} \operatorname{tr}\left(V_{\mu \nu} V^{\mu \nu}\right)+x_{4} m_{\rho}^{2} \operatorname{tr}\left(\hat{\chi}+\hat{\chi}^{\dagger}\right),
\end{aligned}
$$




$$
\begin{aligned}
\mathcal{L}_{4}^{y}= & y_{1} \operatorname{tr}\left(\left(\hat{\alpha}_{\mu \perp} \hat{\alpha}_{\perp}^{\mu}\right)^{2}\right)+y_{2} \operatorname{tr}\left(\hat{\alpha}_{\mu \perp} \hat{\alpha}_{\nu \perp} \hat{\alpha}_{\perp}^{\mu} \hat{\alpha}_{\perp}^{\nu}\right)+y_{3} \operatorname{tr}\left(\left(\hat{\alpha}_{\mu \|} \hat{\alpha}_{\|}^{\mu}\right)^{2}\right)+y_{4} \operatorname{tr}\left(\hat{\alpha}_{\mu \|} \hat{\alpha}_{\nu \|} \hat{\alpha}_{\|}^{\mu} \hat{\alpha}_{\|}^{\nu}\right) \\
& +y_{5} \operatorname{tr}\left(\hat{\alpha}_{\mu \perp} \hat{\alpha}_{\perp}^{\mu} \hat{\alpha}_{\nu \|} \hat{\alpha}_{\|}^{\nu}\right)+y_{6} \operatorname{tr}\left(\hat{\alpha}_{\mu \perp} \hat{\alpha}_{\nu \perp} \hat{\alpha}_{\|}^{\mu} \hat{\alpha}_{\|}^{\nu}\right) \\
& +y_{7} \operatorname{tr}\left(\hat{\alpha}_{\mu \perp} \hat{\alpha}_{\nu \perp} \hat{\alpha}_{\|}^{\nu} \hat{\alpha}_{\|}^{\mu}\right)+y_{8}\left[\operatorname{tr}\left(\hat{\alpha}_{\mu \perp} \hat{\alpha}_{\|}^{\mu} \hat{\alpha}_{\nu \perp} \hat{\alpha}_{\|}^{\nu}\right)+\operatorname{tr}\left(\hat{\alpha}_{\mu \|} \hat{\alpha}_{\perp}^{\mu} \hat{\alpha}_{\nu \|} \hat{\alpha}_{\perp}^{\nu}\right)\right] \\
& +y_{9} \operatorname{tr}\left(\hat{\alpha}_{\mu \perp} \hat{\alpha}_{\nu \|} \hat{\alpha}_{\perp}^{\mu} \hat{\alpha}_{\|}^{\nu}\right) \\
& +y_{10}\left(\operatorname{tr}\left(\hat{\alpha}_{\mu \perp} \hat{\alpha}_{\perp}^{\mu}\right)\right)^{2}+y_{11} \operatorname{tr}\left(\hat{\alpha}_{\mu \perp} \hat{\alpha}_{\nu \perp}\right) \operatorname{tr}\left(\hat{\alpha}_{\perp}^{\mu} \hat{\alpha}_{\perp}^{\nu}\right)+y_{12}\left(\operatorname{tr}\left(\hat{\alpha}_{\mu \|} \hat{\alpha}_{\|}^{\mu}\right)\right)^{2} \\
& +y_{13} \operatorname{tr}\left(\hat{\alpha}_{\mu \|} \hat{\alpha}_{\nu \|}\right) \operatorname{tr}\left(\hat{\alpha}_{\|}^{\mu} \hat{\alpha}_{\|}^{\nu}\right)+y_{14} \operatorname{tr}\left(\hat{\alpha}_{\mu \perp} \hat{\alpha}_{\perp}^{\mu}\right) \operatorname{tr}\left(\hat{\alpha}_{\nu \|} \hat{\alpha}_{\|}^{\nu}\right)+y_{15} \operatorname{tr}\left(\hat{\alpha}_{\mu \perp} \hat{\alpha}_{\nu \perp}\right) \operatorname{tr}\left(\hat{\alpha}_{\|}^{\mu} \hat{\alpha}_{\|}^{\nu}\right) \\
& +y_{16}\left(\operatorname{tr}\left(\hat{\alpha}_{\mu \perp} \hat{\alpha}_{\|}^{\mu}\right)\right)^{2}+y_{17} \operatorname{tr}\left(\hat{\alpha}_{\mu \perp} \hat{\alpha}_{\nu \|}\right) \operatorname{tr}\left(\hat{\alpha}_{\perp}^{\mu} \hat{\alpha}_{\|}^{\nu}\right)+y_{18} \operatorname{tr}\left(\hat{\alpha}_{\mu \perp} \hat{\alpha}_{\nu \|}\right) \operatorname{tr}\left(\hat{\alpha}_{\|}^{\mu} \hat{\alpha}_{\perp}^{\nu}\right), \\
\mathcal{L}_{4}^{z}= & z_{1} \operatorname{tr}\left(\hat{\mathcal{V}}_{\mu \nu} \hat{\mathcal{V}}^{\mu \nu}\right)+z_{2} \operatorname{tr}\left(\hat{\mathcal{A}}_{\mu \nu} \hat{\mathcal{A}}^{\mu \nu}\right)+z_{3} \operatorname{tr}\left(\hat{\mathcal{V}}_{\mu \nu} V^{\mu \nu}\right) \\
& +z_{4} i \operatorname{tr}\left(V_{\mu \nu} \hat{\alpha}_{\perp}^{\mu} \hat{\alpha}_{\perp}^{\nu}\right)+z_{5} i \operatorname{tr}\left(V_{\mu \nu} \hat{\alpha}_{\|}^{\mu} \hat{\alpha}_{\|}^{\nu}\right)+z_{6} i \operatorname{tr}\left(\hat{\mathcal{V}}_{\mu \nu} \hat{\alpha}_{\perp}^{\mu} \hat{\alpha}_{\perp}^{\nu}\right)+z_{7} i \operatorname{tr}\left(\hat{\mathcal{V}}_{\mu \nu} \hat{\alpha}_{\|}^{\mu} \hat{\alpha}_{\|}^{\nu}\right) \\
& +z_{8} i \operatorname{tr}\left(\hat{\mathcal{A}}_{\mu \nu}\left(\hat{\alpha}_{\perp}^{\mu} \hat{\alpha}_{\|}^{\nu}+\hat{\alpha}_{\|}^{\mu} \hat{\alpha}_{\perp}^{\nu}\right)\right) .
\end{aligned}
$$

The one loop calculations are required to make use of systematic expansion of this kind[面]. We adopt the background field technique[2] to avoid an unnecessary complication of defining off-shell effective fields: Naive calculation without a background field requires redefinitions of the off-shell effective fields to maintain manifest chiral symmetry at the loop level 15. Actually, the "low energy theorem" of the HLS 14 cannot be proven without an appropriate field redefinition in the naive covariant gauge calculation [16]. On the other hand, the background field method maintains manifest symmetry at each step of calculations as we will show in the following.

We introduce background fields $\bar{\xi}_{L}, \bar{\xi}_{R}$ and $\bar{V}_{\mu}$ :

$$
\xi_{L}=\xi_{S} \xi_{P}^{\dagger} \bar{\xi}_{L}, \quad \xi_{R}=\xi_{S} \xi_{P} \bar{\xi}_{R}, \quad V_{\mu}=\bar{V}_{\mu}+v_{\mu}
$$

where dynamical degrees of freedom are denoted by $\xi_{S}, \xi_{P}$ and $v_{\mu}$ :

$$
\xi_{S}=\exp \left(\frac{i u_{S}^{a} T^{a}}{\sqrt{a} f}\right), \quad \xi_{P}=\exp \left(\frac{i u_{P}^{a} T^{a}}{f}\right), \quad v_{\mu}=g v_{\mu}^{a} T^{a} .
$$

Transformation properties under HLS are

$$
\begin{aligned}
\bar{\xi}_{L, R} & \rightarrow h(x) \bar{\xi}_{L, R}, \\
\bar{V}_{\mu} & \rightarrow h(x) \bar{V}_{\mu} h^{\dagger}(x)+i h(x) \partial_{\mu} h^{\dagger}(x), \\
u_{S, P}^{a} T^{a} & \rightarrow h(x) u_{S, P}^{a} T^{a} h^{\dagger}(x), \\
v_{\mu}^{a} T^{a} & \rightarrow h(x) v_{\mu}^{a} T^{a} h^{\dagger}(x) .
\end{aligned}
$$

Note here that the dynamical fields $u_{P}^{a}, u_{S}^{a}$ and $v_{\mu}^{a}$ are transformed linearly. Thus, expansion of the chiral lagrangian in terms of these fields does not violate the HLS.

In this formalism, a gauge fixing term for $v_{\mu}^{a}$ can be introduced without violating the HLS of the background field $\bar{V}_{\mu}$ (background gauge 17, 18)

$$
\mathcal{L}_{\mathrm{GF}}=-\frac{1}{2}\left(\bar{D}^{a b \mu} v_{\mu}^{b}+m_{\rho} u_{S}^{a}\right)^{2}
$$


with $\bar{D}_{\mu}$ being the "covariant derivative" on the background field:

$$
\bar{D}^{a b \mu} v_{\mu}^{b} T^{a} \equiv \partial^{\mu} v_{\mu}^{a} T^{a}-i\left[\bar{V}^{\mu}, v_{\mu}^{a} T^{a}\right] .
$$

The Faddeev-Popov determinant associated with the gauge fixing term (16) is

$$
\mathcal{L}_{\mathrm{FP}}=i \bar{c}^{a}\left(\bar{D}^{a b \mu} \bar{D}_{\mu}^{b c}+m_{\rho}^{2} \delta^{a c}\right) c^{c}+\cdots,
$$

where $\cdots$ stands for the interaction terms of the dynamical fields $u_{S}^{a}$, $v_{\mu}^{a}$ and the FP ghosts.

Now, it is straightforward to evaluate the one loop contribution to the above $\mathcal{O}\left(E^{4}\right)$ lagrangian coefficients. After tedious manipulation in the heat kernel method[2], we obtain coefficients of the logarithmic divergences in the dimensional regularization scheme ast

$$
w_{i}=w_{i}^{r}-\frac{\Gamma_{w i}}{4}\left[\frac{\Gamma(2-d / 2)}{(4 \pi)^{d / 2}}+\frac{1}{(4 \pi)^{2}}\right],
$$

and similarly for the coefficients $x_{i}, y_{i}$ and $z_{i}$. The result is summarized in table 1 . These coefficients determine the running of the renormalized parameters,

$$
\mu \frac{\partial}{\partial \mu} w_{i}^{r}(\mu)=-\frac{\Gamma_{w i}}{2(4 \pi)^{2}}, \quad \cdots,
$$

which can be considered as a measure of the effect of radiative corrections.

It should be noted, however, these renormalization group equations (20) are derived in the mass independent scheme and thus the scale $\mu$ is not directly related to the actual physics scale, especially at the low energy region below $m_{\rho}$ where the dynamical degree of freedom of the $\rho$ meson is frozen out. Actually, as we will show in the following, finite part of the one loop integral is important to make the matching conditions with the conventional ChPT without the $\rho$ meson. To construct effective couplings valid in the whole energy region we find it convenient to introduce the *-functions in analogous way with those defined in the electroweak effective lagrangian [19].

The ${ }^{*}$-functions are functions of momentum and independent of renormalization schemes. They are designed to reproduce the real part of the scattering amplitude at the one loop level by replacing the tree level parameters. Such functions can be easily calculated once the effective action is determined. For example, the ${ }^{*}$-functions corresponding to $f, a$ and $g$ are calculated by extracting the corresponding terms from the real part of the effective action:

$$
\begin{aligned}
\Re \mathrm{e} \Gamma= & \int d^{4} x d^{4} z \tilde{f}_{*}^{2}(z) \operatorname{tr}\left(\hat{\alpha}_{\mu \perp}(x+z) \hat{\alpha}_{\perp}^{\mu}(x)\right) \\
& +\int d^{4} x d^{4} z \tilde{a}_{*}(z) f^{2} \operatorname{tr}\left(\hat{\alpha}_{\mu \|}(x+z) \hat{\alpha}_{\|}^{\mu}(x)\right) \\
& -\int d^{4} x d^{4} z \frac{1}{2 \tilde{g}_{*}^{2}(z)} \operatorname{tr}\left(V_{\mu \nu}(x+z) V^{\mu \nu}(x)\right) \cdots,
\end{aligned}
$$

\footnotetext{
${ }^{2}$ Renormalization scale independent quadratic divergences can be renormalized by the redefinition of parameters in the $\mathcal{O}\left(E^{2}\right)$ lagrangian.
} 
with $\tilde{f}_{*}^{2}, \tilde{a}_{*}$ and $1 / \tilde{g}_{*}^{2}$ being Fourier transform of ${ }^{*}$-functions $f_{*}^{2}\left(p^{2}\right), a_{*}\left(p^{2}\right)$ and $1 / g_{*}^{2}\left(p^{2}\right)$, respectively. These ${ }^{*}$-functions can be determined from the two-point functions of the building blocks $\hat{\alpha}_{\mu \perp}, \hat{\alpha}_{\mu \|}, \ldots$ In the chiral limit, we find:

$$
\begin{aligned}
f_{*}^{2}\left(p^{2}\right)= & f^{2}+m_{\rho}^{2}\left\{x_{1}^{r}\left(m_{\rho}\right)+\frac{N a}{(4 \pi)^{2}}\left[1+B_{0}\left(1,0 ; \frac{-p^{2}}{m_{\rho}^{2}}\right)-\frac{1}{4} B_{2}\left(1,0 ; \frac{-p^{2}}{m_{\rho}^{2}}\right)\right]\right\}, \quad \text { (21a) } \\
a_{*}\left(p^{2}\right) f^{2}= & a f^{2}+m_{\rho}^{2}\left\{x_{2}^{r}\left(m_{\rho}\right)+\frac{N}{(4 \pi)^{2}}\left[1+B_{0}\left(1,1 ; \frac{-p^{2}}{m_{\rho}^{2}}\right)\right]\right\}, \\
\frac{1}{g_{*}^{2}\left(p^{2}\right)}= & \frac{1}{g^{2}}-2 x_{3}^{r}\left(m_{\rho}\right) \\
& +\frac{N}{(4 \pi)^{2}}\left[-\frac{a^{2}}{24} \Re \operatorname{e} \ln \left(\frac{-p^{2}}{m_{\rho}^{2}}\right)+\frac{31}{24}+\frac{5 a^{2}}{72}+4 B_{0}\left(1,1 ; \frac{-p^{2}}{m_{\rho}^{2}}\right)-\frac{9}{8} B_{4}\left(1,1 ; \frac{-p^{2}}{m_{\rho}^{2}}\right)\right],
\end{aligned}
$$

with $B_{0}, B_{2}$ and $B_{4}$ being defined by:

$$
\begin{aligned}
& B_{0}(X, Y ; P) \equiv \Re \mathrm{e} \int_{0}^{1} d \alpha \ln (\alpha X+(1-\alpha) Y+\alpha(1-\alpha) P), \\
& B_{2}(X, Y ; P) \equiv \Re \mathrm{e} \int_{0}^{1} d \alpha(1-2 \alpha) \ln (\alpha X+(1-\alpha) Y+\alpha(1-\alpha) P), \\
& B_{4}(X, Y ; P) \equiv \Re \mathrm{e} \int_{0}^{1} d \alpha(1-2 \alpha)^{2} \ln (\alpha X+(1-\alpha) Y+\alpha(1-\alpha) P) .
\end{aligned}
$$

The typical size of $\mathcal{O}\left(E^{4}\right)$ corrections can be estimated in this calculation. Expanding Eq. $(21 \mathrm{~b})$ around $p^{2}=0$, we obtain

$$
a_{*}\left(p^{2}\right) f^{2}-a_{*}(0) f^{2}=-\frac{1}{6} \frac{N}{(4 \pi)^{2}} p^{2}+\mathcal{O}\left(\frac{p^{4}}{m_{\rho}^{2}}\right)
$$

and find $a_{*}\left(p^{2}\right)$ is insensitive to the loop effect. Actually, $a_{*}\left(p^{2}\right)$ receives only $10 \%$ level radiative correction: $a_{*}\left(m_{\rho}^{2}\right)=a_{*}(0)-0.17$ in $S U(2)$ model for $f=88 \mathrm{MeV}$.

We can extract information of $\mathcal{O}\left(E^{4}\right)$ parameters through the matching conditions with the conventional $\mathrm{ChPT}$ without the $\rho$ meson which is already studied extensively by Gasser and Leutwyler 2]. Gasser-Leutwyler lagrangian is given by

$$
\begin{aligned}
\mathcal{L}_{\mathrm{GL}}= & \frac{F^{2}}{4} \operatorname{tr}\left(\left(D_{\mu} U\right)^{\dagger}\left(D^{\mu} U\right)\right)+\frac{F^{2}}{4} \operatorname{tr}\left(U^{\dagger} X+X^{\dagger} U\right) \\
& +L_{1}\left(\operatorname{tr}\left(\left(D_{\mu} U\right)^{\dagger}\left(D^{\mu} U\right)\right)\right)^{2}+\cdots \\
& -i L_{9} \operatorname{tr}\left(\mathcal{R}_{\mu \nu}\left(D^{\mu} U\right)^{\dagger}\left(D^{\nu} U\right)+\mathcal{L}_{\mu \nu}\left(D^{\mu} U\right)\left(D^{\nu} U\right)^{\dagger}\right) \\
& +L_{10} \operatorname{tr}\left(U^{\dagger} \mathcal{L}_{\mu \nu} U \mathcal{R}^{\mu \nu}\right)+H_{1} \operatorname{tr}\left(\mathcal{L}_{\mu \nu} \mathcal{L}^{\mu \nu}+\mathcal{R}_{\mu \nu} \mathcal{R}^{\mu \nu}\right)+H_{2} \operatorname{tr}\left(X^{\dagger} X\right),
\end{aligned}
$$

with $X=2 B(s+i p)$ and $F$ being the NG boson decay constant in the chiral limit. In the following, we focus our attention on the parameters $L_{9,10}$. It is convenient to 
introduce the ${ }^{*}$-functions corresponding to the parameters $L_{9,10}$ and $H_{1}$. In the chiral limit, we find

$$
\begin{aligned}
F_{*}^{2}\left(p^{2}\right) & =F^{2}, \\
L_{9}^{*}\left(p^{2}\right) & =L_{9}^{r}-\frac{N}{24(4 \pi)^{2}}\left(-\frac{5}{3}+\Re \mathrm{e} \ln \frac{-p^{2}}{\mu^{2}}\right), \\
L_{10}^{*}\left(p^{2}\right) & =L_{10}^{r}+\frac{N}{24(4 \pi)^{2}}\left(-\frac{5}{3}+\Re \mathrm{e} \ln \frac{-p^{2}}{\mu^{2}}\right), \\
H_{1}^{*}\left(p^{2}\right) & =H_{1}^{r}+\frac{N}{48(4 \pi)^{2}}\left(-\frac{5}{3}+\Re \mathrm{e} \ln \frac{-p^{2}}{\mu^{2}}\right) .
\end{aligned}
$$

To obtain the matching conditions, we integrate out $\hat{\alpha}_{\mu \|}$ and $V_{\mu \nu}$ from the effective action by making use of the equations of motion:

$$
\hat{\alpha}_{\mu \|}=\frac{1}{m_{\rho}^{2}} \mathcal{O}\left(E^{3}\right)
$$

and

$$
V_{\mu \nu}=\hat{\mathcal{V}}_{\mu \nu}+i\left[\hat{\alpha}_{\mu \perp}, \hat{\alpha}_{\nu \perp}\right]+\frac{1}{m_{\rho}^{2}} \mathcal{O}\left(E^{4}\right) .
$$

The latter equation can be derived from

$$
D_{\mu} \hat{\alpha}_{\nu \|}-D_{\nu} \hat{\alpha}_{\mu \|}-i\left[\hat{\alpha}_{\mu \|}, \hat{\alpha}_{\nu \|}\right]=\frac{1}{m_{\rho}^{2}} \mathcal{O}\left(E^{4}\right),
$$

and Eq.(9b). Thus, we obtain:

$$
\begin{gathered}
F_{*}^{2}\left(p^{2}\right)=f_{*}^{2}\left(p^{2}\right)+\mathcal{O}\left(\frac{p^{2}}{m_{\rho}^{2}}\right), \\
-8 L_{9}^{*}\left(p^{2}\right)=-\frac{2}{g_{*}^{2}\left(p^{2}\right)}+2 z_{3}^{*}\left(p^{2}\right)+z_{4}^{*}\left(p^{2},-p^{2}, 0\right) \\
+z_{6}^{*}\left(p^{2},-p^{2}, 0\right)+\mathcal{O}\left(\frac{p^{2}}{m_{\rho}^{2}}\right), \\
L_{10}^{*}\left(p^{2}\right)+2 H_{1}^{*}\left(p^{2}\right)=-\frac{1}{2 g_{*}^{2}\left(p^{2}\right)}+z_{1}^{*}\left(p^{2}\right)+z_{3}^{*}\left(p^{2}\right)+\mathcal{O}\left(\frac{p^{2}}{m_{\rho}^{2}}\right), \\
-L_{10}^{*}\left(p^{2}\right)+2 H_{1}^{*}\left(p^{2}\right)=z_{2}^{*}\left(p^{2}\right)+\frac{1}{2} f_{*}^{2 \prime}(0)+\mathcal{O}\left(\frac{p^{2}}{m_{\rho}^{2}}\right) .
\end{gathered}
$$

The ${ }^{*}$-functions corresponding to $z_{1}, z_{2}$ and $z_{3}$ are determined as $z_{1}^{*}\left(p^{2}\right)=z_{1}^{r}\left(m_{\rho}\right)+\frac{N}{16(4 \pi)^{2}}\left[\frac{(2-a)^{2}}{3} \Re \operatorname{eln}\left(\frac{-p^{2}}{m_{\rho}^{2}}\right)-\frac{5}{9}(2-a)^{2}+\frac{1}{3}+B_{4}\left(1,1 ; \frac{-p^{2}}{m_{\rho}^{2}}\right)\right]$, 


$$
\begin{aligned}
& z_{2}^{*}\left(p^{2}\right)=z_{2}^{r}\left(m_{\rho}\right)+\frac{N a}{8(4 \pi)^{2}}\left[\frac{1}{3}+B_{4}\left(1,0 ; \frac{-p^{2}}{m_{\rho}^{2}}\right)\right] \\
& z_{3}^{*}\left(p^{2}\right)=z_{3}^{r}\left(m_{\rho}\right)+\frac{N}{8(4 \pi)^{2}}\left[\frac{a(2-a)}{3} \Re \ln \left(\frac{-p^{2}}{m_{\rho}^{2}}\right)-\frac{5}{9} a(2-a)+\frac{1}{3}+B_{4}\left(1,1 ; \frac{-p^{2}}{m_{\rho}^{2}}\right)\right] .
\end{aligned}
$$

The ${ }^{*}$-functions corresponding to $z_{4}$ and $z_{6}$ depend not simply on single momentum, since they lead to non-oblique three point vertices of $\rho-\pi-\pi$ and photon- $\pi-\pi$, respectively:

$$
z_{4}^{*}\left(p^{2}, q^{2}, p \cdot q\right), \quad z_{6}^{*}\left(p^{2}, q^{2}, p \cdot q\right),
$$

where $p$ and $q$ stand for incoming momentum of $\rho$ (photon) and the difference of two incoming pion momenta, respectively. These ${ }^{*}$-functions become simple when we take $q^{2}=-p^{2}, p \cdot q=0$ (corresponding to the on-shell of pions in the 3-point vertex):

$$
\begin{aligned}
z_{4}^{*}\left(p^{2},-p^{2}, 0\right)= & z_{4}^{r}\left(m_{\rho}\right)+\frac{N}{4(4 \pi)^{2}}\left[\frac{a(2-a)}{3} \Re \operatorname{e} \ln \left(\frac{-p^{2}}{m_{\rho}^{2}}\right)-\frac{5}{9} a(2-a)+\frac{1}{3}\right. \\
& \left.+B_{4}\left(1,1 ; \frac{-p^{2}}{m_{\rho}^{2}}\right)-a C\left(1,0 ; \frac{-p^{2}}{m_{\rho}^{2}}\right)-a^{2} C\left(0,1 ; \frac{-p^{2}}{m_{\rho}^{2}}\right)\right], \\
z_{6}^{*}\left(p^{2},-p^{2}, 0\right)= & z_{6}^{r}\left(m_{\rho}\right)+\frac{N}{4(4 \pi)^{2}}\left[\frac{(2-a)^{2}}{3} \Re \operatorname{en}\left(\frac{-p^{2}}{m_{\rho}^{2}}\right)-\frac{5}{9}(2-a)^{2}+\frac{1}{3}\right. \\
& \left.+B_{4}\left(1,1 ; \frac{-p^{2}}{m_{\rho}^{2}}\right)-a C\left(1,0 ; \frac{-p^{2}}{m_{\rho}^{2}}\right)-a(2-a) C\left(0,1 ; \frac{-p^{2}}{m_{\rho}^{2}}\right)\right]
\end{aligned}
$$

with $C$ being defined by

$$
C(X, Y ; P) \equiv \frac{1}{6}+\Re \mathrm{e} \int_{0}^{1} d \alpha \int_{0}^{1} d \beta \alpha(2 \alpha-1) \ln \left(\alpha X+(1-\alpha) Y+\alpha^{2} \beta(1-\beta) P\right) .
$$

It should be stressed here that the infrared singularity $\left(\ln \left(p^{2}\right)\right)$ in $z_{i}^{*}(i=1,2,3,4,6)$ does not appear at $a=2$ (vector dominance).

Note here that the coefficients of $\ln \left(p^{2}\right)$ arise from the finite part of the one loop integral and agree exactly in both sides of Eq.(24). It should also be stressed that the matching conditions (24) are free from the renormalization scale ambiguity in contrast with the previous tree level analysis of the resonance effect to Gasser-Leutwyler parameters 10.

By using the values of $S U(2)$ Gasser-Leutwyler parameters 22, 10] $L_{9}^{r}(550 \mathrm{MeV})=$ $(7.2 \pm 0.7) \cdot 10^{-3}, L_{10}^{r}(550 \mathrm{MeV})=(-5.5 \pm 0.3) \cdot 10^{-3}$ and $f=f_{*}(0)=88 \mathrm{MeV}, m_{\rho}=$ $757 \mathrm{MeV}$ estimated in the chiral limit, we find a cancellation among $L_{9,10}^{*}, 1 / g_{*}^{2}$ and $f_{*}^{2 \prime}$ in (24) and obtain:

$$
\frac{1}{2}\left(z_{1}^{*}(0)-z_{2}^{*}(0)+z_{3}^{*}(0)\right)=(-0.6 \pm 0.3) \cdot 10^{-3},
$$




$$
\frac{1}{8}\left(2 z_{3}^{*}(0)+z_{4}^{*}(0,0,0)+z_{6}^{*}(0,0,0)\right)=(0.7 \pm 0.7) \cdot 10^{-3}
$$

or

$$
\begin{aligned}
\frac{1}{2}\left(z_{1}^{*}\left(m_{\rho}^{2}\right)-z_{2}^{*}\left(m_{\rho}^{2}\right)+z_{3}^{*}\left(m_{\rho}^{2}\right)\right) & =(0.1 \pm 0.3) \cdot 10^{-3}, \\
\frac{1}{8}\left(2 z_{3}^{*}\left(m_{\rho}^{2}\right)+z_{4}^{*}\left(m_{\rho}^{2},-m_{\rho}^{2}, 0\right)+z_{6}^{*}\left(m_{\rho}^{2},-m_{\rho}^{2}, 0\right)\right) & =(0.8 \pm 0.7) \cdot 10^{-3},
\end{aligned}
$$

where we have assumed the vector dominance $a=a_{*}(0)=2$ and used

$$
g_{*}^{2}\left(m_{\rho}^{2}\right)=\frac{m_{\rho}^{2}}{a_{*}\left(m_{\rho}^{2}\right) f^{2}} .
$$

There is no strong indication for the presence of the non-minimal couplings $z_{i}^{*}$. In other words, the Gasser-Leutwyler estimates for $L_{9,10}$ are saturated by the $\rho$ meson and its one loop contribution without introducing non-minimal $\mathcal{O}\left(E^{4}\right)$ terms. This result contrasts with the tree level analysis indicating that $L_{10}$ is not saturated by the $\rho$ meson contribution alone but saturated by the combined effect of the $\rho$ meson and the $a_{1}$ meson 10. Actually, the tree level contribution from the $a_{1}$ resonance is imitated by the one loop effect $f_{*}^{2 \prime}$ in the matching conditions (24).

In this paper, we have formulated the ChPT of the $\pi$ - $\rho$ system based on the effective lagrangian [4 with the hidden local symmetry. The size of the radiative correction to the phenomenological parameter $a$ is estimated to be about $10 \%$. We also obtain the matching conditions to the conventional ChPT of Gasser-Leutwyler in the renormalization scale independent manner. It should be emphasized that the one loop effect imitates the tree level contribution of the $a_{1}$ resonance in the matching condition for $L_{10}$. We find that Gasser-Leutwyler's estimates for $L_{9,10}$ are saturated by the $\rho$ meson and its one loop contribution without introducing the $\mathcal{O}\left(E^{4}\right)$ couplings.

Technical details of the present work will be published elsewhere.

The author thanks K. Yamawaki for introducing him to the importance of this subject. He is very grateful to Y. Okada for continuous encouragements and fruitful discussions. He thanks also K. Hagiwara, M. Harada, M. Kobayashi, T. Kugo, S. Matsumoto, V.A. Miransky, A.I. Sanda, M. Tanaka and T.N. Truong for enlightening discussions. 


\section{References}

[1] S. Weinberg, Physica 96A (1979) 327.

[2] J. Gasser and H. Leutwyler, Ann. Phys. (N.Y.) 158 (1984) 142; Nucl. Phys. B250 (1985) 465.

[3] B. Holdom and J. Terning, Phys. Lett. B247 (1990) 88; M. Golden and L. Randall, Nucl. Phys. B361 (1991) 3; H. Georgi, Nucl. Phys. B363 (1991) 301; A.F. Falk, M. Luke, E.H. Simmons, Nucl. Phys. B365 (1991) 523.

[4] M. Bando, T. Kugo, S. Uehara, K. Yamawaki and T. Yanagida, Phys. Rev. Lett. 54 (1985) 1215; For a review, see M. Bando, T. Kugo and K. Yamawaki, Phys. Reports 164 (1988) 218.

[5] S. Weinberg, Phys. Rev. 166 (1968) 1568.

[6] J.J. Sakurai, Currents and Mesons (Univ. Chicago Press, Chicago, 1969).

[7] K. Kawarabayashi and M. Suzuki, Phys. Rev. Lett. 16 (1966) 255; Riazuddin and Fayyazuddin, Phys. Rev. 147 (1966) 1071.

[8] H. Georgi, Phys. Rev. Lett. 63 (1989) 1917; Nucl. Phys. B331 (1990) 311.

[9] P. Ko, Phys. Rev. D44 (1991) 139; Phys. Rev. D46 (1992) 3813; M. Finkemeier, Phys. Rev. D47 (1993) 1933.

[10] G. Ecker, J. Gasser, H. Leutwyler, A. Pich and E. de Rafael, Phys. Lett. B223 (1989) 425. See also G. Ecker, J. Gasser, A. Pich and E. de Rafael, Nucl. Phys. B321 (1989) 311; J.F. Donoghue, C. Ramirez and G. Valencia, Phys. Rev. D39 (1989) 1947.

[11] R. Casalbuoni, S. de Curtis, D. Dominici and R. Gatto, Phys. Lett. B155, (1985) 95; Nucl. Phys. B282, (1987) 235.

[12] G. Cvetič and R. Kögerler, Nucl. Phys. B363 (1991) 401.

[13] M. Harada and K. Yamawaki, Phys. Lett. B297 (1992) 151.

[14] M. Bando, T. Kugo and K. Yamawaki, Nucl. Phys. B259 (1985) 493; Prog. Theor. Phys. 73 (1985) 1541.

[15] T. Appelquist and C. Bernard, Phys. Rev. D23 (1981) 425.

[16] M. Harada, T. Kugo and K. Yamawaki, Nagoya preprint DPNU-93-01; Kyoto preprint KUNS-1179 HE(TH) 93/02.

[17] For a review, see L.F. Abbott, Acta. Phys. Pol. B13 (1982) 33. 
[18] Yu.N. Kafiev, Nucl. Phys. B201 (1982) 341.

[19] D.C. Kennedy and B.W. Lynn, Nucl. Phys. B322 (1989) 1. 


\begin{tabular}{|c|c|c|c|}
\hline & $\overline{\Gamma_{w i}}$ & $a=1$ & $a=2$ \\
\hline$w_{1}$ & $2 N(4-3 a)$ & $2 N$ & $-4 N$ \\
\hline$w_{2}$ & $2(4-3 a)$ & 2 & -4 \\
\hline$w_{3}$ & $2 N a^{2}$ & $2 N$ & $8 N$ \\
\hline$w_{4}$ & $2 a^{2}$ & 2 & 8 \\
\hline$w_{5}$ & $\frac{3}{2} N a(1-a)$ & 0 & $-3 N$ \\
\hline$w_{6}$ & $\frac{2}{N}\left(N^{2}-4\right)$ & $\frac{2}{N}\left(N^{2}-4\right)$ & $\frac{2}{N}\left(N^{2}-4\right)$ \\
\hline$w_{7}$ & $\frac{2}{N^{2}}\left(N^{2}+2\right)$ & $\frac{2}{N^{2}}\left(N^{2}+2\right)$ & $\frac{2}{N^{2}}\left(N^{2}+2\right)$ \\
\hline$w_{8}$ & $-N a$ & $-N$ & $-2 N$ \\
\hline \multirow[t]{2}{*}{$w_{9}$} & $a$ & 1 & 2 \\
\hline & $\overline{\Gamma_{x i}}$ & $a=1$ & $a=2$ \\
\hline$x_{1}$ & $-3 N a$ & $-3 N$ & $-6 N$ \\
\hline$x_{2}$ & $-3 N$ & $-3 N$ & $-3 N$ \\
\hline$x_{3}$ & $\frac{N}{12}\left(87-a^{2}\right)$ & $\frac{43 N}{6}$ & $\frac{83 N}{12}$ \\
\hline$x_{4}$ & 0 & 0 & 0 \\
\hline & $\overline{\Gamma \Gamma_{z i}}$ & $a=1$ & $a=2$ \\
\hline $\begin{array}{l}z_{1} \\
z_{2} \\
z_{3} \\
z_{4} \\
z_{5} \\
z_{6} \\
z_{7} \\
z_{8}\end{array}$ & $\begin{array}{c}-\frac{N}{12}\left(5-4 a+a^{2}\right) \\
-\frac{N}{6} a \\
-\frac{N}{6}\left(1+2 a-a^{2}\right) \\
-\frac{N}{6}\left(2+3 a-3 a^{2}\right) \\
-\frac{N}{6}\left(1+2 a^{2}-a^{3}\right) \\
-\frac{N}{6}(2-a)(5-3 a) \\
-\frac{N}{6}\left(1+4 a-4 a^{2}+a^{3}\right) \\
\frac{N}{6} a(1+a) \\
\end{array}$ & $\begin{array}{l}-\frac{N}{6} \\
-\frac{N}{6} \\
-\frac{N}{3} \\
-\frac{N}{3} \\
-\frac{N}{3} \\
-\frac{N}{3} \\
-\frac{N}{3} \\
\frac{N}{3}\end{array}$ & $\begin{array}{l}-\frac{N}{12} \\
-\frac{N}{3} \\
-\frac{N}{6} \\
\frac{2 N}{3} \\
-\frac{N}{6} \\
0 \\
-\frac{N}{6} \\
N \\
\end{array}$ \\
\hline
\end{tabular}




\begin{tabular}{|l||c|c|c|}
\hline & $\Gamma_{y i}$ & $a=1$ & $a=2$ \\
\hline$y_{1}$ & $\frac{N}{6}\left(7-11 a+5 a^{2}\right)$ & $\frac{N}{6}$ & $\frac{5 N}{6}$ \\
$y_{2}$ & $\frac{N}{12}\left(10-14 a+5 a^{2}\right)$ & $\frac{N}{12}$ & $\frac{N}{6}$ \\
$y_{3}$ & $\frac{N}{12}\left(1-2 a^{2}+2 a^{3}+a^{4}\right)$ & $\frac{N}{6}$ & $\frac{25 N}{12}$ \\
$y_{4}$ & $\frac{N}{24}\left(1+4 a^{2}-4 a^{3}+a^{4}\right)$ & $\frac{N}{12}$ & $\frac{N}{24}$ \\
$y_{5}$ & $\frac{N}{6} a\left(1+6 a-5 a^{2}\right)$ & $\frac{N}{3}$ & $-\frac{7 N}{3}$ \\
$y_{6}$ & $\frac{N}{6}\left(1+4 a-5 a^{2}+2 a^{3}\right)$ & $\frac{N}{3}$ & $\frac{5 N}{6}$ \\
$y_{7}$ & $\frac{N}{6}\left(-1+10 a-13 a^{2}+6 a^{3}\right)$ & $\frac{N}{3}$ & $\frac{5 N}{2}$ \\
$y_{8}$ & $\frac{N}{6} a^{2}(4-3 a)$ & $\frac{N}{6}$ & $-\frac{4 N}{3}$ \\
$y_{9}$ & $\frac{N}{6} a^{2}$ & $\frac{N}{6}$ & $\frac{2 N}{3}$ \\
$y_{10}$ & $\frac{1}{4}\left(8-12 a+5 a^{2}\right)$ & $\frac{1}{4}$ & 1 \\
$y_{11}$ & $\frac{1}{2}\left(8-12 a+5 a^{2}\right)$ & $\frac{1}{2}$ & 2 \\
$y_{12}$ & $\frac{1}{8}\left(1+a^{4}\right)$ & $\frac{1}{2}$ & $\frac{17}{8}$ \\
$y_{13}$ & $\frac{1}{4}\left(1+a^{4}\right)$ & $\frac{1}{2}$ & $\frac{17}{4}$ \\
$y_{14}$ & $\frac{1}{6} a\left(1+7 a-5 a^{2}\right)$ & 1 & $\frac{5}{3}$ \\
$y_{15}$ & $\frac{1}{3} a\left(7-5 a+a^{2}\right)$ & 1 & $\frac{2}{3}$ \\
$y_{16}$ & $\frac{1}{3} a\left(7-5 a+a^{2}\right)$ & 1 & $-\frac{10}{3}$ \\
$y_{17}$ & $\frac{1}{3} a\left(1+7 a-5 a^{2}\right)$ & 1 & $\frac{2}{3}$ \\
$y_{18}$ & $\frac{1}{3} a\left(7-5 a+a^{2}\right)$ & \\
\hline
\end{tabular}

Table 1: Coefficients of counter terms 